\title{
Altered amygdala effective connectivity in migraine without aura: evidence from resting-state fMRI with Granger causality analysis
}

Xiaobin Huang ${ }^{1}$, Di Zhang ${ }^{1}$, Peng Wang ${ }^{1}$, Cunnan Mao ${ }^{1}$, Zhengfei Miao ${ }^{1}$, Chunmei Liu², Chenjie Xu ${ }^{3}$, Xindao Yin ${ }^{1}$ and Xinying $\mathrm{Wu}^{1 *}$

\begin{abstract}
Background: Granger causality analysis (GCA) has been used to investigate the pathophysiology of migraine. Amygdala plays a key role in pain modulation of migraine attack. However, the detailed neuromechanism remained to be elucidated. We applied GCA to explore the amygdala-based directional effective connectivity in migraine without aura (MwOA) and to determine the relation with clinical characteristics.

Methods: Forty-five MwoA patients and forty age-, sex-, and years of education-matched healthy controls(HCs) underwent resting-state functional magnetic resonance imaging (fMRI). Bilateral amygdala were used as seed regions in GCA to investigate directional effective connectivity and relation with migraine duration or attack frequency.
\end{abstract}

Results: MwoA patients showed significantly decreased effective connectivity from right amygdala to right superior temporal gyrus, left superior temporal gyrus and right precentral gyrus compared with HCs. Furthermore, MwoA patients demonstrated significantly decreased effective connectivity from the left amygdala to the ipsilateral superior temporal gyrus. Also, MwoA patients showed enhanced effective connectivity from left inferior frontal gyrus to left amygdala. Effective connectivity outflow from right amygdala to right precentral gyrus was negatively correlated to disease duration.

Conclusions: Altered directional effective connectivity of amygdala demonstrated that neurolimbic pain networks contribute to multisensory integration abnormalities and deficits in pain modulation of MwoA patients.

Keywords: Migraine, Granger causality analysis, Amygdala, Pain modulation

\section{Introduction}

Migraine is a disabling primary headache disorder and was ranked third-highest cause of disability worldwide in both males and females under the age of 50 years[1]. Migraine without aura(MwoA) is the most common type

\footnotetext{
* Correspondence: rebeccahxt@163.com

'Department of Radiology, Nanjing First Hospital, Nanjing Medical University, No.68, Changle Road Nanjing, 210006 Nanjing, Jiangsu Province, China Full list of author information is available at the end of the article
}

of migraine. It is a recurrent headache disorder menifesting in attacks lasting $4-72 \mathrm{~h}$ with typically unilateral in location, pulsating in quality, moderate to severe in intensity, aggravated by physical activity, and associated with nausea and light and sound sensitivity (photophobia and phonophobia) $[2,3]$. However, the neuromechanism of the MwoA, which would help to choose optimal treatment to improve the life quality of patients, is remaining elucidated. 
Amygdala, which is a large grey matter complex in neurolimbic system, plays a key role in pain modulation during a migraine attack[4-7]. Aberrant connectivities between amygdala and somatosensory cortex regions in migraineurs were observed[8]. Seed-based whole-brain correlation method showed migraine patients have disrupted limbic system (amygdala and hippocampus) functional connectivity to pain-related cortex regions of modulatory and encoding[9]. However, most of previous studies about the effective connectivity in neurolimbic system omit the directional influence of the cerebral functional cortex on amygdala. The directional influence of amygdala might reflect pain modulation principle in MwoA. Fortunately, Granger causality analysis (GCA) is an advanced fMRI data processing method to investigate the top-down control between the cerebral functional cortex and the amygdala [10-12]. The specific intrinsic brain effective connectivity among pain-related networks in MwoA patients are also affected after long-term migraine attacks[13, 14]. Though many studies have proved that migraine is associated with changes in functional connectivity between different regions, no studies discern the directionality or specificity of the disrupted connections related to amygdala.

To address this issue, we applied GCA to identify differences in the direction of functional connectivity between MwoA patients and healthy controls. We selected the bilateral amygdala as seed regions to elucidate mechanism of neurolimbic pain-modulation in migraine pathogenesis. We hypothesized that GCA and functional connectivity of these regions would be disrupted in migraine patients. Moreover, these alterations would be associated with clinical characteristics such as disease duration or attack frequency. To our knowledge, this is the first study to use GCA to unravel the effective connectivity within the limbic system in migraine patients.

\section{Method \\ Participants}

According to the International Classification of Headache Disorders, Third Edition (beta version) (ICHD3beta), 45 MwoA patients were recruited in this study between May 2018 and June 2020. All subjects were right-handed. None of the patients took any preventive medications. To avoid possible interference of pain or pharmacological substances on BOLD signal fluctuation, patients were migraine free for at least 3 days before the fMRI scan. The exclusion criteria included psychotic disorder, major physical illness, e.g. cancer, psychoactive, preventive or chronic medication and contraindications to MRI. Meanwhile, 40 age-, sex-, and years of education-matched healthy subjects were recruited as healthy controls (HCs). The inclusion criteria were having no personal or family history of migraine or any other type of headaches, and free from any chronic medical condition. To minimize hormonal influences on cortical excitability, all female participants were included at mid-cycle and excluded if pregnant or breast-feeding. All participants completed the Hamilton Anxiety Scale (HAMA), Hamilton Depression Scale (HAMD), Montreal Cognitive Assessment (MoCA), Headache Impact Test-6 (HIT-6), and Migraine Disability Assessment Questionnaire. This study was approved by the Institutional Review Board of our university. All participants provided written informed consent before undergoing MR scan.

\section{Imaging methods}

A 3.0 Tesla magnetic resonance imaging scanner (Ingenia, Philips Medical Systems, Netherlands) with an 8channel head coil was used in this study. Functional images were obtained axially using a gradient echo-planar imaging sequence as follows: repetition time $(\mathrm{TR})=$ $2000 \mathrm{~ms}$; echo time $(\mathrm{TE})=30 \mathrm{~ms}$; slices $=36$; thickness $=4$ $\mathrm{mm}$; gap $=0 \mathrm{~mm}$; field of view $(\mathrm{FOV})=240 \mathrm{~mm} \times 240$ $\mathrm{mm}$; acquisition matrix $=64 \times 64$; and flip angle $(\mathrm{FA})=$ $90^{\circ}$. The fMRI sequence took $8 \mathrm{~min}$ and $8 \mathrm{~s}$. Threedimensional turbo fast-echo (3D-TFE) $\mathrm{T}_{1} \mathrm{WI}$ sequence with high resolution: $\mathrm{TR}=8.1 \mathrm{~mm} ; \mathrm{TE}=3.7 \mathrm{~ms}$; slices $=$ 170; thickness $=1 \mathrm{~mm}$; gap $=0 \mathrm{~mm}$; FA $=8^{\circ}$; acquisition matrix $=256 \times 256$; FOV $=256 \mathrm{~mm} \times 256 \mathrm{~mm}$; Fluidattenuated inversion recovery (FLAIR): TR $=7000 \mathrm{~ms}$; $\mathrm{TE}=120 \mathrm{~ms}$; slices $=18$; slice thickness $=6 \mathrm{~mm}$; gap $=1.3$ $\mathrm{mm} ; \mathrm{FA}=110^{\circ}$; voxel size $=0.65 \times 0.95 \times 6 \mathrm{~mm}^{3}$.

\section{Image processing}

Data analyses were preprocessed using Data Processing Assistant for Resting-State fMRI programs[15], which is based on Statistical Parametric Mapping (SPM8, http:// www.fil.ion.ucl.ac.uk/spm) and resting-state fMRI data analysis toolkit (REST, http://www.restfmri.net). The first 10 volumes were discarded and the remaining 230 consecutive volumes were used for data analysis. Slicetiming and realignment for head motion correction were performed. Subjects with a head motion greater than 2.0 $\mathrm{mm}$ translation or a $2.0^{\circ}$ rotation in any direction were excluded. Data were spatially normalized to the Montreal Neurological Institute template (resampling voxel size $=3 \times 3 \times 3 \mathrm{~mm}^{3}$ ), smoothed with an isotropic Gaussian kernel [full width at half maximum $(\mathrm{FWHM})=4$ $\mathrm{mm}]$, detrended and filtered $(0.01-0.08 \mathrm{~Hz})$. The bilateral amygdala were set as seed regions using the WFU_ PickAtlas software (http://www.ansir.wfubmc.edu). Effective connectivity was analyzed using REST-GCA in the REST toolbox [16]. In this study, time series of bilateral amygdala were defined as seed time series $\mathrm{x}$, and time series y denoted all voxels in the brain. The linear direct influence of $\mathrm{x}$ on $\mathrm{y}(\mathrm{F} \mathrm{x} \rightarrow \mathrm{y})$ and the linear direct 
Table 1 Demographic characteristics of the patients with MwoA and the healthy controls

\begin{tabular}{llll}
\hline & MwoA patients $(\boldsymbol{n}=\mathbf{4 5})$ & HCs $(\boldsymbol{n}=\mathbf{4 0})$ & $\boldsymbol{P}$ value \\
\hline Age(years) & $38.62 \pm 10.11$ & $35.45 \pm 7.53$ & 0.108 \\
Gender(male/female) & $12: 33$ & $14: 26$ & 0.482 \\
MoCA score & $27 \pm 1.03$ & $27.73 \pm 1.93$ & 0.069 \\
HAMA score & $37.16 \pm 8.68$ & $35.5 \pm 6.78$ & 0.334 \\
HAMD score & $41.76 \pm 8.93$ & $40.60 \pm 7.92$ & 0.532 \\
Education(years) & $14.36 \pm 2.72$ & $15.03 \pm 2.38$ & 0.233 \\
Duration(years) & $13.8 \pm 6.07$ & $\mathrm{NA}$ & $\mathrm{NA}$ \\
Frequency(d/m) & $4.31 \pm 4.34$ & $\mathrm{NA}$ & $\mathrm{NA}$ \\
HIT-6 score & $58.80 \pm 8.09$ & $\mathrm{NA}$ & $\mathrm{NA}$ \\
MIDAS score & $12.29 \pm 6.95$ & $\mathrm{NA}$ & $\mathrm{NA}$ \\
\hline
\end{tabular}

Data are presented as mean \pm SD; MwoA Migraine without aura; HCs Healthy controls; MoCA Montreal Cognitive Assessment; HAMA Hamilton Anxiety Scale; HAMD Hamilton Depression Scale; $d / m$ day per month; HIT-6 Headache Impact Test-6; MIDAS Migraine Disability Assessment Questionnaire

influence of $y$ on $x(F \quad y \rightarrow x)$ were calculated voxel by voxel across the brain. Afterwards, two Granger causality maps were generated based on the influence measures for each subject. The residual-based $F$ was normalized $\left(F^{\prime}\right)$ and standardized to $Z$ score for each voxel $\left(Z_{x \rightarrow y}\right.$ and $Z_{y \rightarrow x}$, subtracting the global mean F'values, divided by standard deviation).

\section{Statistical analysis}

For group level analyses on effective connectivity of amygdala, mean values of $\mathrm{Zx} \rightarrow \mathrm{y}$ and $\mathrm{Zy} \rightarrow \mathrm{x}$ maps were calculated for each group. All eight Granger causality maps were acquired, with four for each direction and four for each group (left amygdala with $Z x \rightarrow y$ and $\mathrm{Zy} \rightarrow \mathrm{x}$ and right amygdala with $\mathrm{Zx} \rightarrow \mathrm{y}$ and $\mathrm{Zy} \rightarrow \mathrm{x}$ for both the patients and healthy controls). These Granger causality maps were entered into a voxel-wise twosample t-test to determine the difference between MwoA patients and HCs with age, sex, and education included as covariates. For multiple comparison correction, Gaussian Random Field (GRF) with $p<0.01$ (Z $>$ 2.58) for voxel level results and $p<0.05$ for cluster level was used. The threshold of cluster size was set at 118 voxels. Positive clusters based on RESTplus were generated as binary mask, and connective strengths of the significant regions were extracted based on z-maps.

Comparison of demographic data between MwoA patients and $\mathrm{HCs}$ were analyzed by using between-group $\mathrm{t}$-test for means and 2-test for proportions ( $p<0.05$ was considered to be significant). To investigate the relation between fMRI data and clinical cognitive characteristic of MwoA, regions showing significant difference between groups were extracted. Then the mean z-values of aberrant functional connectivity region mask were calculated within each subject. Pearson's correlation analysis between mean $\mathrm{z}$-values of aberrant functional connectivity and each clinical cognitive characteristic were performed by using SPSS 17.0 (version 17.0; SPSS, Chicago, IL, USA). $P<0.05$ was considered as statistically significant, corrected for age, sex and years of education. Bonferroni correction was used for multiple comparisons in correlation analyses.

\section{Results \\ Demographic and clinical variables}

As shown in Table 1, MwoA patients and HCs showed no significant difference in gender $(P=0.482)$, age $(P=$ $0.108)$, MoCA $\operatorname{score}(P=0.069)$, HAMA score $(P=0.334)$, HAMD $\operatorname{score}(P=0.532)$, or years of education $(P=$ $0.233)$.

\section{Granger causality analysis}

MwoA patients showed significantly decreased effective connectivity from right amygdala to several brain regions that include right superior temporal gyrus, left superior temporal gyrus and right precentral gyrus compared with $\mathrm{HCs}$ (Table 2; Fig. 1). Furthermore, MwoA patients demonstrated significantly decreased effective connectivity from the left amygdala to the ipsilateral superior temporal gyrus (Table 3; Fig. 2). Also, MwoA patients showed enhanced effective connectivity from left inferior frontal gyrus to left amygdale (Table 3; Fig. 3).

Table 2 Two-sample t-test (voxel-level $P<0.01$ and cluster-level $P<0.05$ Gaussian random field corrected) of difference in causal influence to and from the right amygdala in patients with migraine without aura versus healthy controls

\begin{tabular}{|c|c|c|c|c|c|c|}
\hline & \multirow[t]{2}{*}{ Brain region } & \multicolumn{3}{|c|}{ Peak MNI coordinates } & \multirow{2}{*}{$\begin{array}{l}\text { Voxel } \\
\text { size }\end{array}$} & \multirow{2}{*}{$\begin{array}{l}\text { Peak t } \\
\text { score }\end{array}$} \\
\hline & & $\mathrm{x}$ & $\mathrm{Y}$ & $\mathbf{Z}$ & & \\
\hline \multicolumn{7}{|l|}{ Causal outflow from $R$ amygdala to the rest of the brain ( $X$ to $Y$ ) } \\
\hline & L_Sup_Temporal & -66 & -48 & 12 & 214 & -4.7341 \\
\hline & R_Sup_Temporal & 54 & -12 & -6 & 366 & -4.3447 \\
\hline & R_Precentral & 42 & -3 & 45 & 136 & -4.9252 \\
\hline
\end{tabular}

Causal inflow to $R$ amygdala from the rest of the brain ( $Y$ to $X)$ 


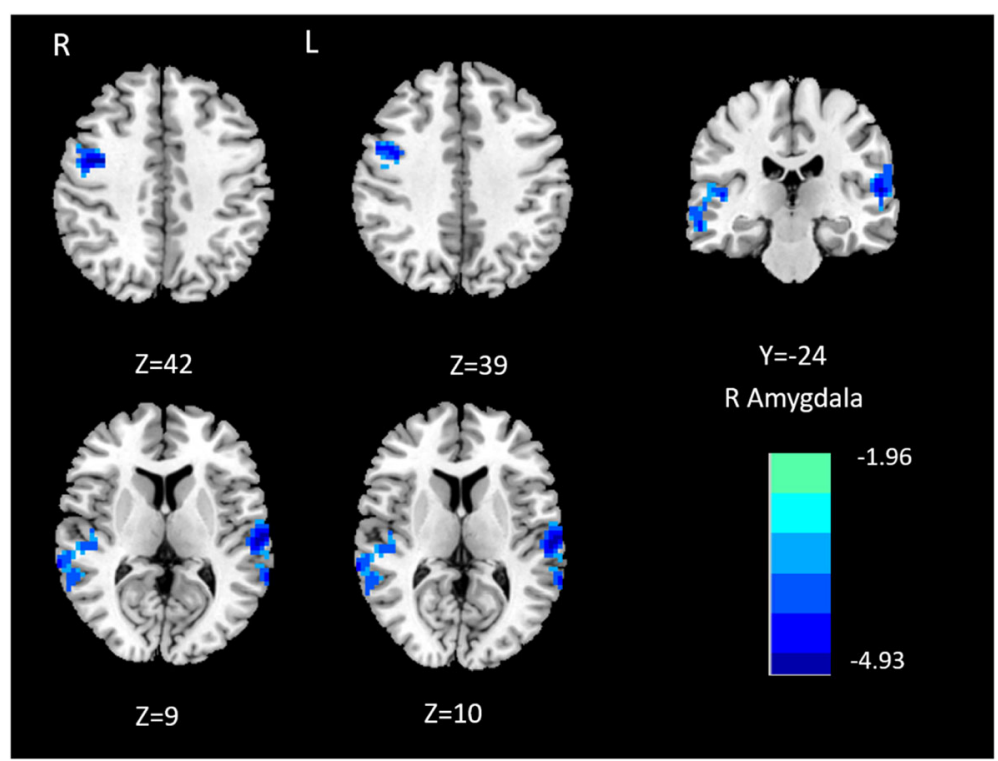

Fig. 1 Causal outflow from $R$ amygdala to the rest of the brain ( $X$ to $Y$ )

\section{Correlation analysis}

Effective connectivity outflow from right amygdala to right precentral gyrus was negatively correlated to disease duration (Fig. 4, $P=0.003, r=-0.429$ ). No other significant linear correlation was observed.

\section{Discussion}

The relation between limbic system and pain always receives much concern[17-19]. As an essential element of the limbic system, amygdala is worth of further study in migraineurs. To our knowledge, this is the first study to use GCA to identify changes in effective connectivity (directional connectivity) in amygdala which is strongly implicated in migraine. Our main finding showed that MwoA patients had significant changes in effective connectivity in a set of brain regions including diverse functional areas emanating from and projecting to the amygdale.

Our study indicated that there is a significantly decreased effective connectivity from the right amygdala to the bilateral superior temporal gyrus. During interictal period, MwoA patients might still suffer hypersensitivity to auditory, olfactory, or visual stimuli. The superior temporal gyrus is generally considered to be important as auditory perception and emotional regulatory part in human brain[20-23], which is essential for individual stressful experiences, cognitive processes and adaptive behavior. The amygdala is capable of modulating auditory cortex activity and plasticity that allows a particular individual to respond to the environment in a predictive manner. Some previous studies have shown that migraineurs, in the interictal phase and in migraine attack, have abnormalities in the function of neural substrates, responsible for different stages of temporal processing, especially in temporal resolution and temporal ordering[24-27]. Our results of decreased connectivity in each hemisphere from amygdala to superior temporal gyrus in MwoA patients may represent temporal processing impairment, which could be a reasonable explanation for photophobia and phonophobia linked to lower threshold for tolerating unpleasant signals. The superior temporal gyrus plays other neurocognitive functions, such as speaking and language recognition. In our study, MwoA

Table 3 Two-sample t-test (voxel-level $P<0.01$ and cluster-level $P<0.05$ Gaussian random field corrected) of difference in causal influence to and from the left amygdala in patients with migraine without aura versus healthy controls

\begin{tabular}{|c|c|c|c|c|c|c|}
\hline & \multirow[t]{2}{*}{ Brain region } & \multicolumn{3}{|c|}{ Peak MNI coordinates } & \multirow{2}{*}{$\begin{array}{l}\text { Voxel } \\
\text { size }\end{array}$} & \multirow{2}{*}{$\begin{array}{l}\text { Peak t } \\
\text { score }\end{array}$} \\
\hline & & $\mathbf{x}$ & $\mathrm{Y}$ & $\mathbf{Z}$ & & \\
\hline \multicolumn{7}{|l|}{ Causal outflow from $L$ amygdala to the rest of the brain ( $X$ to $Y$ ) } \\
\hline & L_Sup_Temporal & -60 & -42 & 12 & 203 & -3.5682 \\
\hline \multicolumn{7}{|l|}{ Causal inflow to $L$ amygdala from the rest of the brain ( $Y$ to $X$ ) } \\
\hline & L_Inf_Frontal & -39 & -15 & -24 & 118 & 6.0593 \\
\hline
\end{tabular}




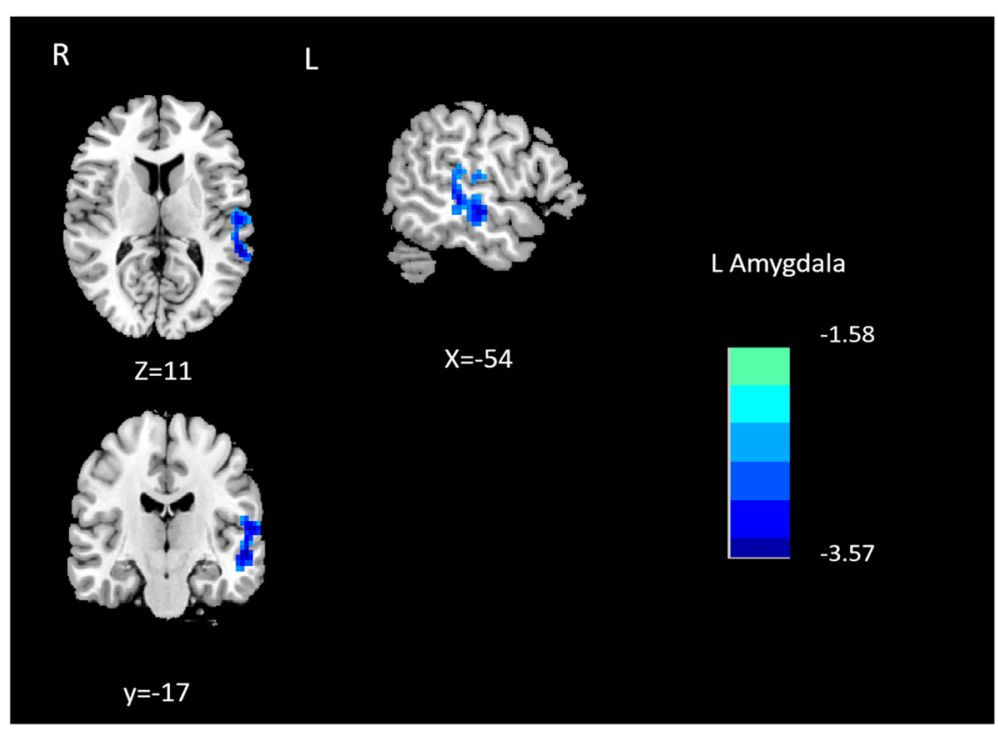

Fig. 2 Causal outflow from $L$ amygdala to the rest of the brain ( $X$ to $Y$ )

patients showed decreased scores of visuospatial and executive function, name recognition and language compared with HCs (Table 4). Decreased superior temporal gyrus connectivity represented naming and language defects to some extent.

Migraine patients also showed decreased functional connectivity from the left amygdala to the left superior temporal gyrus. This laterality pattern may be associated with the different functions of bilateral amygdala, and the left amygdala was known to contribute to the brain's reward system[28]. Therefore, the laterality mechanism should be further investigated.

Another finding of the present study was the decreased connectivity from the right amygdala to the right precentral gyrus. The effective connectivity outflow from right amygdala to the right precentral gyrus was negatively correlated with migraine duration. The precentral gyrus, which is part of the supplementary motor area(SMA), predominantly participants in the internally generated planning of movement, the planning of sequences of movement, also involved in pain anticipation. The

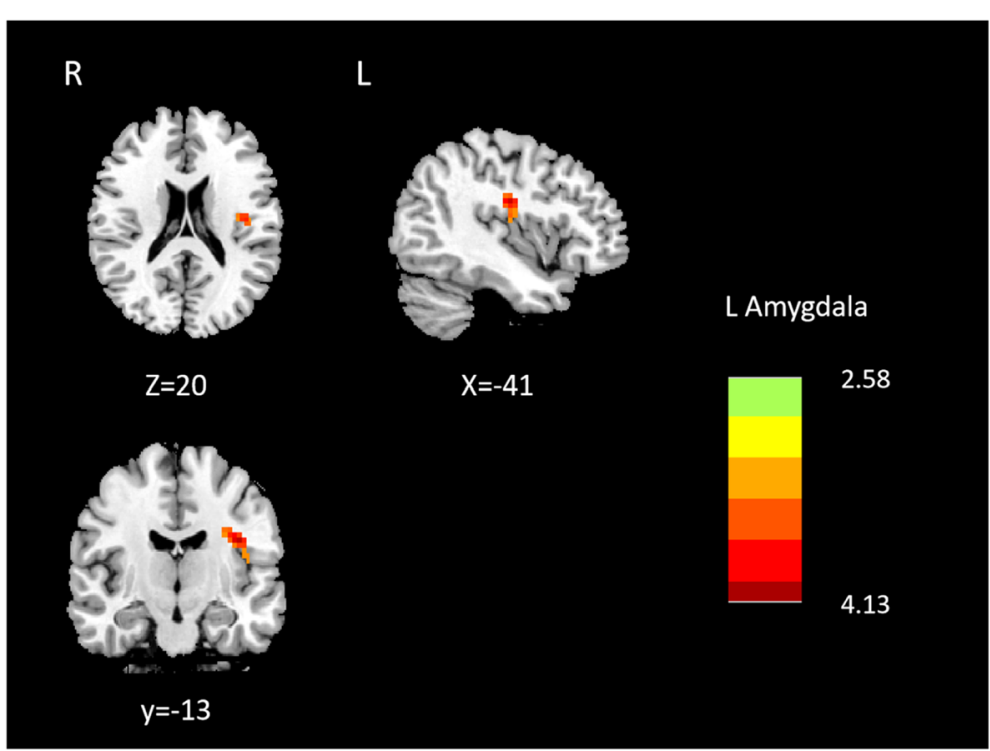

Fig. 3 Causal inflow to $L$ amygdala from the rest of the brain ( $Y$ to $X)$ 
Table 4 Detailed category statistical analysis according to MoCA

\begin{tabular}{lccc}
\hline & MwoA patients $(\mathbf{n}=\mathbf{4 5})$ & Healthy controls $(\mathbf{n}=\mathbf{4 0})$ & $P$ value \\
\hline Visuospatial and executive function & $2.37 \pm 0.451$ & $4.67 \pm 0.867$ & 0.021 \\
Name recognition & $1.21 \pm 0.532$ & $2.95 \pm 0.284$ & 0.014 \\
Attention & $5.77 \pm 0.721$ & $5.85 \pm 0.495$ & 0.432 \\
Language & $1.81 \pm 0.412$ & $2.92 \pm 0.195$ & 0.032 \\
Abstract & $1.79 \pm 0.431$ & $1.93 \pm 0.503$ & 0.851 \\
Delayed memory & $3.45 \pm 1.369$ & $3.73 \pm 1.325$ & 0.654 \\
Orientation & $5.81 \pm 0.507$ & $5.97 \pm 0.313$ & 0.221 \\
\hline
\end{tabular}

reduced connectivity from the right amygdala to right precentral gyrus may be associated with disrupted projections and modulation of pain perception[17]. Since physical movement aggaravated the pain of migraine, less activation of precentral gyrus in MwoA at interictal period might indicate protective adaptive plasticity alteration after repetitive movement related pain experience. Our findings of reduced connectivity within SMA are in line with those of other studies showing decreased regional homogeneity and cortical thinning in migraineurs[29-31]. We speculate that nociceptive input modifies the functional patterns of the SMA and that these changes may account for the functional impairment in migrainuers.

Inferior frontal gyrus not only plays a role in cognitive modulation of pain, but also is involved in memory retrieval and emotional pain regulation [32]. Previous studies also showed aberrant cerebral perfusion changes[33] in the inferior frontal gyrus in migraineurs. Prior resting-state fMRI study also provided further support linking migraine physiopathology with the inferior frontal gyrus[34]. The enhanced effective connectivity from left inferior frontal gyrus to left amygdala might indicate maladaptive brain response due to repeated stress or unpleasant exposure in migraineurs. As such, migraine can be regarded as the consequence of multisensory interactions between pain modulation and limbic network.

Our study has several potential limitations. First, the moderate small sample size may have reduced our ability to detect causal relationships between abnormal effective connectivity and migraine characteristics. Furthermore, we examined patients only in the interictal phase, and therefore effective connectivity in the ictal phase must also be explored. Finally, the seed used in this study covered the whole amygdala; however the amygdala was subdivided into the basolateral nucleus group and the medial cortex group. Different parts may have their own distinct impact; therefore more accurately defined anatomical

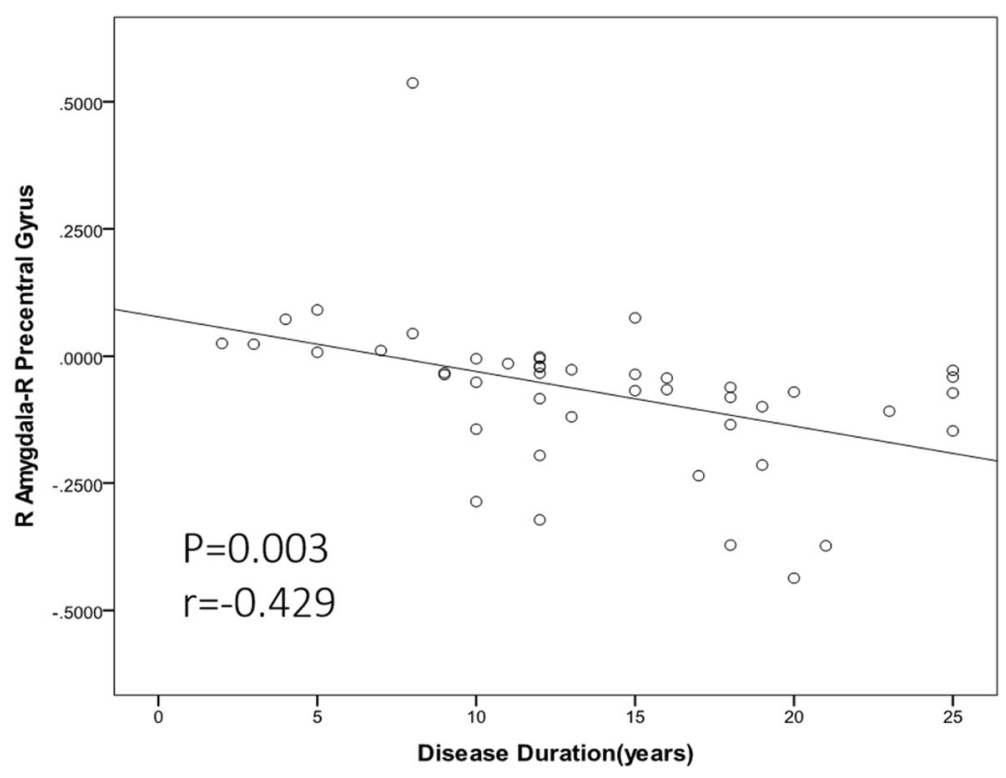

Fig. 4 Correlations between effective connectivity from right amygdala to right precentral gyrus and disease duration 
parcellation may influence the final results. These issues will be addressed in subsequent studies.

\section{Conclusions}

Despite these limitations, our results identified disrupted effective connectivity networks in the amygdala of migraine patients. These findings may be associated with multisensory integration abnormalities and deficits in pain modulation that play a key role in the clinical characteristics in migraineurs, which could help enhance our understanding of the neuropathological mechanism underlying migraine.

\section{Acknowledgements}

We would like to thank all the migraine patients and healthy controls who participated in our research.

\section{Authors' contributions}

XW designed the study. XH, DZ, WS, YC, PW, CM, ZM, CL and CX performed the experiments. $\mathrm{XH}$ analysed the data and were major contributor in writing the manuscript. All authors read and approved the final manuscript.

\section{Funding}

This work was supported by Nanjing Science and Technology Planning Project (No. 202002056).

\section{Availability of data and materials}

All data and materials generated in this study are available upon request.

\section{Declarations}

\section{Ethical approval and consent to participate}

This study was conducted in accordance with the ethical standards of the institutional and/or national research committee and with the 1964 Helsinki declaration. All participants gave their informed, written consent.

\section{Consent for publication}

Not applicable.

\section{Competing interests}

The authors declare that they have no competing interests.

\section{Author details}

'Department of Radiology, Nanjing First Hospital, Nanjing Medical University, No.68, Changle Road Nanjing, 210006 Nanjing, Jiangsu Province, China. ${ }^{2}$ Department of Neurology, Nanjing First Hospital, Nanjing Medical University, No.68, Changle Road, 210006 Nanjing, Jiangsu Province, China. ${ }^{3}$ Department of Pain Treatment, Nanjing First Hospital, Nanjing Medical University, No.68, Changle Road, 210006 Nanjing, Jiangsu Province, China.

Received: 22 January 2021 Accepted: 5 April 2021

Published online: 15 April 2021

\section{References}

1. Ducros A, Arnold M, Dichgans M, Houdart E, Leroux J et al (2013). The International Classification of Headache Disorders, 3rd edition (beta version). Cephalalgia, 33(9): 629-808.

2. Aurora SK, Shrewsbury SB, Ray S, Hindiyeh N, Nguyen L (2021)A link between gastrointestinal disorders and migraine: Insights into the gut-brain connection. Headache doi:https://doi.org/10.1111/head.14099

3. Miglis, Mitchell G (2018) Migraine and Autonomic Dysfunction: Which Is the Horse and Which Is the Jockey?. Curr Pain Headache Rep, 22(3):19

4. Maleki N, Becerra L, Borsook D (2012) Migraine: maladaptive brain responses to stress. Headache, 2012, 52(Suppl. 2):102-106

5. Schwedt, Todd J (2013) Multisensory integration in migraine. Curr Opin Neurol, 26(3):248-253
6. Hadjikhani N, Ward N, Boshyan J, Napadow V, Maeda Y et al (2013) The missing link: enhanced functional connectivity between amygdala and visceroceptive cortex in migraine. Cephalalgia 33(15): 1264

7. Schulte LH, Menz MM, Haaker J, May A. (2020) The migraineur's brain networks: Continuous resting state fMRI over 30 days. Cephalalgia 40(14):1614-1621

8. Chen Z, Chen X, Liu M, Dong Z, Ma L et al (2017) Altered functional connectivity of amygdala underlying the neuromechanism of migraine pathogenesis. J Headache Pain, 18(1)

9. Wei H, Chen J, Chen Y, Yu Y, Zhang, H (2019). Impaired functional connectivity of limbic system in migraine without aura. Brain Imaging Behav, 14(5): 1805-1814

10. Jiang Y, Tian Y, Wang Z (2019) Causal Interactions in Human Amygdala Cortical Networks across the Lifespan. Sci Rep 9(1):5927

11. Wang M, Zeng N, Zheng H, Du X, Potenza MN, Dong GH. (2020) Altered effective connectivity from the pregenual anterior cingulate cortex to the laterobasal amygdala mediates the relationship between internet gaming disorder and loneliness. Psychol Med 20:1-10

12. Mao N, Che K, Xie H, Li Y, Wang Q, Liu M, et al (2020) Abnormal information flow in postpartum depression: A resting-state functional magnetic resonance imaging study. J Affect Disord 277:596-602

13. Zheng L, Yang G. F, Zhang XY, Wang Y. F, Liu Y et al (2017) Altered amygdala and hippocampus effective connectivity in mild cognitive impairment patients with depression: a resting-state functional mr imaging study with granger causality analysis. Oncotarget, 8(15).

14. Chen F, Ke J, Qi R, Xu Q, Zhong Y et al (2018). Increased Inhibition of the Amygdala by the mPFC may Reflect a Resilience Factor in Post-traumatic Stress Disorder: A Resting-State fMRI Granger Causality Analysis. Front Psychiatry, 9: 516

15. Yan C, Zang Y (2010) DPARSF: a MATLAB toolbox for "pipeline" data analysis of resting-state fMRI. Front Syst Neurosci, 4: 13

16. Zang ZX, Yan C. G, Dong ZY, Huang J, Zang YF (2012) Granger causality analysis implementation on matlab: a graphic user interface toolkit for fmri data processing. J Neurosci Methods, 203(2): 418-426

17. Androulakis XM, Rorden C, Peterlin BL, Krebs K. (2018) Modulation of salience network intranetwork resting state functional connectivity in women with chronic migraine. Cephalalgia 38(11):1731-1741

18. Tonini, M.C. (2018)Gender differences in migraine. Neurol Sci 39, 77-78.

19. Chong CD, Schwedt TJ, Hougaard A (2019)Brain functional connectivity in headache disorders: A narrative review of MRI investigations. J Cereb Blood Flow Metab.39(4):650-669

20. Resnik J, Paz R (2015) Fear generalization in the primate amygdala. Nat Neurosci, 18(2): 188-190

21. Michelle M, Federico D M, Elia F (2014) An anatomical and functional topography of human auditory cortical area. Front Neurosci, 8(8):225

22. Schoenwiesner M, Zatorre R J (2009) Spectro-temporal modulation transfer function of single voxels in the human auditory cortex measured with highresolution fMRI[J]. Proc Nati Acad Sci U S A, 106(34):14611-14616

23. Yi HG, Leonard M. K, Chang EF (2019) The Encoding of Speech Sounds in the Superior Temporal Gyrus. Neuron, 102(6): 1096-1110

24. Schwedt TJ (2014) Chronic migraine. BMJ 348:g1416

25. Zeyher C, Frerichs O (2017) Auditory Processing in Children with Migraine: A Controlled Study. Neuropediatrics, 48(2): 123-126

26. Agessi L, Villa Thaís, Dias K, Carvalho D. D, Pereira L (2014). Central auditory processing and migraine: a controlled study. J Headache Pain, 15: 72

27. Wei HL, Chen YC, Yu YS, Guo X, Zhou GP, Zhou QQ et al (2021) Aberrant activity within auditory network is associated with psychiatric comorbidities in interictal migraineurs without aura. Brain Imaging Behav. doi: https:/doi.org/10.1007/s11682-020-00446-9

28. Korostenskaja M, Pardos M, Kujala T, Rose D. F, Hershey AD (2011). Impaired auditory information processing during acute migraine: a magnetoencephalography study. Int J Neurosci, 121(7): 355-365

29. Chen C, Yan M, Yu Y, Ke J, Zhao H et al (2019) Alterations in Regional Homogeneity Assessed by fMRI in Patients with Migraine Without Aura. J Med Syst, 43(9): 298

30. Zhang Y, Chen H, Zeng M, He J, Qi G, Zhang S, et al. (2020) Abnormal Whole Brain Functional Connectivity Pattern Homogeneity and Couplings in Migraine Without Aura. Front Hum Neurosci. 11;14:619839

31. Qin Z, Su J, He XW, Ban S, Zhu Q, Cui Y, et al. (2020) Disrupted functional connectivity between sub-regions in the sensorimotor areas and cortex in migraine without aura. J Headache Pain. 6;21(1):47

32. Ochsner KN, Silvers J A, Buhle JT (2012) Functional imaging studies of emotion regulation: $A$ synthetic review and evolving model of the cognitive control of emotion. Ann N Y Acad Sci, 1251: E1-E24 
33. Arkink EB, Bleeker E. J, Schmitz N, Schoonman G. G, Kruit MC et al (2012) Cerebral perfusion changes in migraineurs: a voxelwise comparison of interictal dynamic susceptibility contrast MRI measurements. Cephalalgia, 32(4): 279-288

34. Zhang D, Huang X, Su W, Chen Y, Wu X et al (2020). Altered lateral geniculate nucleus functional connectivity in migraine without aura: a resting-state functional MRI study. J Headache Pain, 21(1): 17

\section{Publisher's Note}

Springer Nature remains neutral with regard to jurisdictional claims in published maps and institutional affiliations.

Ready to submit your research? Choose BMC and benefit from:

- fast, convenient online submission

- thorough peer review by experienced researchers in your field

- rapid publication on acceptance

- support for research data, including large and complex data types

- gold Open Access which fosters wider collaboration and increased citations

- maximum visibility for your research: over $100 \mathrm{M}$ website views per year

At $B M C$, research is always in progress.

Learn more biomedcentral.com/submissions 